\title{
Les enceintes urbaines et villageoises de la Loire
}

Prospection thématique (2013)

\section{Jacques Verrier}

\section{(2) OpenEdition \\ Journals}

Édition électronique

URL : https://journals.openedition.org/adlfi/15328

ISSN : 2114-0502

Éditeur

Ministère de la Culture

Référence électronique

Jacques Verrier, «Les enceintes urbaines et villageoises de la Loire » [notice archéologique], ADLFI. Archéologie de la France - Informations [En ligne], Auvergne-Rhône-Alpes, mis en ligne le 11 août 2015, consulté le 23 juillet 2021. URL : http://journals.openedition.org/adlfi/15328

Ce document a été généré automatiquement le 23 juillet 2021.

(c) ministère de la Culture et de la Communication, CNRS 


\title{
Les enceintes urbaines et villageoises de la Loire
}

Prospection thématique (2013)

\author{
Jacques Verrier
}

\section{NOTE DE L'ÉDITEUR}

Organisme porteur de l'opération : Groupe de recherches archéologiques de la Loire

1 L'étude pour l'année 2013 sur les enceintes urbaines et villageoises a concerné quatre sites : une campagne de prospection à Saint-Étienne; une étude complète sur l'enceinte de Saint-Priest-en-Jarez et sur celle supposée de Saint-Jean-Bonnefonds; et une étude sur l'enceinte villageoise de Poncins.

2 La campagne de prospection sur l'enceinte urbaine de la ville de Saint-Étienne a montré qu'il en existe encore aujourd'hui des éléments observables, souvent ténus ou difficiles d'accès mais présents. La conservation partielle d'une tour d'angle polygonale située au nord-est et le tracé d'une seconde tour d'angle polygonale au sud-est, inscrit dans le parcellaire, sont des éléments nouveaux et importants. Les vestiges observés d'une partie de la porte de Furan, mis en valeur dans l'Hôtel de la Tour, sont aussi des observations très positives concernant l'enceinte urbaine de la ville.

La présence d'une petite enceinte urbaine à Saint-Priest-en-Jarez, attenante à l'enceinte, n'était à ce jour pas clairement établie. Comme c'est souvent le cas dans les textes anciens, le château et l'habitat fortifié se retrouvaient sous l'appellation plus ou moins générique de castrum, sans qu'il soit possible de les différencier. L'absence de structures clairement identifiables comme des tours (absentes sur le terrain et dans les textes) ou une porte fortifiée (absente sur le terrain mais présente dans les textes) a participé au manque de lisibilité du site. 
D'autres éléments ont amplifié la difficulté :

- la structure du village, en terrasses et soutènements, dans laquelle il est souvent difficile de différencier ce qui appartient à la fortification de ce qui est du domaine de l'aménagement civil ;

- les différents travaux liés à l'usage ou l'usure (effondrements, réfections, modifications), comme d'ailleurs la transformation soupçonnée de la vieille enceinte médiévale en fortification basse.

5 La structure de l'ensemble a tenu compte du relief. Le château et son enceinte sont construits sur le point haut, une plate-forme réaménagée, au nord. Ils profitent de défenses naturelles sur trois côtés, ne laissant que le côté sud pour le développement du village et pour l'accès au château. Cette enceinte villageoise a profité, elle aussi, de la protection offerte par le relief sur les côtés est et ouest. Le côté sud reste le point le plus vulnérable de l'enceinte, à cause d'une pente légèrement plus douce mais surtout en raison de la présence de la porte d'accès, point faible de toute fortification.

D'après les éléments recueillis en archives et en prospection, il semblerait que la fortification ait connu au moins deux périodes. La première va de la création, dont la date est inconnue, jusqu'au $\mathrm{XvI}^{\mathrm{e}} \mathrm{s}$. Il s'agit vraisemblablement d'une structure classique, composée de remparts en élévation suivant au plus près le relief et composant avec lui. La seconde débute au cours $\mathrm{du} \mathrm{XVI}^{\mathrm{e}} \mathrm{s}$. et a consisté à mettre l'ancienne fortification en état de résister aux nouvelles méthodes de guerre et utiliser l'artillerie. Les conséquences sur le village et son enceinte restent du domaine de l'hypothétique. Une fausse braie a été construite en avant et contre l'ancien rempart, au sud.

7 La recherche et la prospection effectuées en 2013 ont permis de dégrossir et de poser quelques bases à la fois sur le château et sur l'enceinte urbaine accolée. Il reste néanmoins beaucoup de questions en suspens et de nombreuses hypothèses attendent une confirmation ou une infirmation. Seul un difficile travail de sondages sur les structures en élévation permettrait peut-être d'avancer dans ce sens.

Les résultats minimalistes des prospections sur l'enceinte de Saint-Jean-Bonnefonds ont confirmé les écrits du XIX ${ }^{e}$ s., notamment ceux de M. de la Tour de Varan, dressant un état assez catastrophique des constructions du bourg à cette époque. Ils laissaient peu d'espoir de retrouver aujourd'hui quelques éléments en place.

9 Actuellement, les seuls arguments tangibles de la présence d'une enceinte urbaine autour de la ville médiévale, sont ceux issus du cadastre napoléonien indiquant la présence de fossés et celle d'un château. L'existence de ce dernier est confirmée par les mentions d'un châtelain, sur les terriers datant des $\mathrm{XVI}^{\mathrm{e}}$ et $\mathrm{XVII}^{\mathrm{e}} \mathrm{s}$.

10 La disposition du parcellaire incite à se demander quelle forme pouvait avoir cette éventuelle enceinte. S'agissait-il d'une association d'habitats juxtaposés formant un simili rempart ou était-ce un rempart, ou encore un vingtain constitué, aujourd'hui totalement ruiné? Les éléments en notre possession tendraient à privilégier la première solution.

11 Les terriers Tinelli et Cuacon ne laissent aucun doute sur la présence d'une petite enceinte villageoise à Poncins au cours des $\mathrm{xv}^{\mathrm{e}}$ et $\mathrm{xvI}^{\mathrm{e}} \mathrm{s}$. Cette fortification reste présente et active assez longtemps puisqu'elle est encore mentionnée en tant qu'ouvrage de défense au XvII ${ }^{\mathrm{e}} \mathrm{s}$.

ADLFI. Archéologie de la France - Informations , Auvergne-Rhône-Alpes 
12 Un tracé hypothétique peut être avancé à l'aide du cadastre napoléonien et de quelques mentions issues principalement du terrier Cuacon de 1534. Cette enceinte de forme ovoïde associe un fossé et un mur de vingtain, que l'on suppose avoir été élevés en pisé. La présence d'une porte, défendue par une tour flanquante, est un élément de fortification important qu'il est difficile d'imaginer aujourd'hui.

13 L'enceinte, mentionnée dans le terrier Tinelli en 1451, est donc antérieure à cette date mais nous ignorons celle de sa création. La disposition des lieux (château au nord, village au sud) telle qu'on la perçoit au travers des différents documents pourrait s'apparenter à l'évolution d'une structure de type château et basse-cour.

14 À partir du $\mathrm{XVI}^{\mathrm{e}}$ s., il semblerait que la volonté du seigneur soit d'évincer toutes ces constructions internes pour les rejeter dans la périphérie de l'enceinte afin d'en conserver l'usage exclusif.

\section{INDEX}

Année de l'opération : 2013

nature https://ark.frantiq.fr/ark:/26678/crtcYIBmBlBPH

chronologie https://ark.frantiq.fr/ark:/26678/pcrtAQyKm9qosx, https://ark.frantiq.fr/ark:/ 26678/pcrtkWqzTusoxz

lieux https://ark.frantiq.fr/ark:/26678/pcrtSEeAipsBlD, https://ark.frantiq.fr/ark:/26678/ crtB8WDyqd6u9, https://ark.frantiq.fr/ark:/26678/pcrtzWwOdr34hl, https://ark.frantiq.fr/ark:/ 26678/pcrtF99kWtypA7, https://ark.frantiq.fr/ark:/26678/pcrtSWBPURfXDT, https:// ark.frantiq.fr/ark:/26678/pcrt9Qa7NXa29w, https://ark.frantiq.fr/ark:/26678/pcrtzGgqvEf1IO sujets https://ark.frantiq.fr/ark:/26678/pcrtKJVpuP3AET, https://ark.frantiq.fr/ark:/26678/ pcrtDlzbGxWvTo, https://ark.frantiq.fr/ark:/26678/pcrtkP8rR1YLpG, https://ark.frantiq.fr/ ark:/26678/pcrt321qD4sfeM, https://ark.frantiq.fr/ark:/26678/pcrtslAx38Lp91, https:// ark.frantiq.fr/ark:/26678/pcrt4otVkR3bqD

\section{AUTEURS}

JACQUES VERRIER

GRAL 